\title{
REFORMA DEL SECTOR SALUD EN EL PERÚ: DERECHO, GOBERNANZA, COBERTURA UNIVERSAL Y RESPUESTA CONTRA RIESGOS SANITARIOS
}

\begin{abstract}
Aníbal Velásquez ${ }^{1,2, a}$, Dalia Suarez ${ }^{2, b}$, Edgardo Nepo-Linares ${ }^{3,4, c}$
RESUMEN

El año 2013 el Perú inició un proceso de reforma bajo la premisa de reconocer que la salud es un derecho y que el Estado debe garantizar su protección. El objetivo fue mejorar el estado de salud buscando eliminar o aminorar las restricciones que le impiden ejercer plenamente este derecho. El enfoque fue el de protección en términos de salud individual, salud pública y de los derechos, en un marco de fortalecimiento de la rectoría y la gobernanza que permitiera conducción del sistema y efectiva respuesta ante riesgos y emergencias. Con la Reforma la cobertura de aseguramiento en la población subió de 64 a 73\% y la universalización se empieza a concretar a través de la afiliación al Seguro Integral de Salud (SIS) de todo recién nacido que no tiene otro mecanismo de protección. El financiamiento en salud se elevó en $75 \%$ comparado con el del año 2011 y el presupuesto del SIS se triplicó, de 570 a 1700 millones de soles. De 2012 a mayo del 2016 se han puesto en operación 168 establecimientos de salud, 51 están por culminar y existen 265 proyectos que están en fase de expediente técnico o continuidad de obra, con una inversión ejecutada de más de 7 mil millones de soles. Con la Reforma se aprobó la intervención del Ministerio de Salud ante emergencias sanitarias y se fortaleció la autoridad sanitaria para que el ministerio implemente respuestas en caso de riesgos o discontinuidad de los servicios por falta de cumplimiento de las funciones de salud pública de los gobiernos regionales o locales.
\end{abstract}

Palabras clave: Reforma de la atención de salud; Cobertura universal; Respuesta a riesgos sanitarios (Fuente: DeCS BIREME).

\section{HEALTH SECTOR REFORM IN PERU: LAW, GOVERNANCE, UNIVERSAL COVERAGE, AND RESPONSES TO HEALTH RISKS}

\begin{abstract}
In 2013, Peru initiated a reform process under the premise of recognizing the nature of health as a right that must be protected by the state. This reform aimed to improve health conditions through the elimination or reduction of restrictions preventing the full exercise of this right, and the consequent approach aimed to protect both individual and public health and rights within a framework characterized by strengthened stewardship and governance, which would allow system conduction and effective responses to risks and emergencies. The reform led to an increase in population health insurance coverage from $64 \%$ to $73 \%$, with universalization occurring through the SIS affiliation of every newborn with no other protection mechanism. Health financing increased by $75 \%$ from 2011 , and the SIS budget tripled from 570 to 1,700 million soles. From 2012 to May 2016, 168 health facilities have become operational, 51 establishments are nearing completion, and 265 new projects are currently under technical file and work continuity with an implemented investment of more than 7 billion soles. Additionally, this reform led to the approval of the Ministry of Health intervention for health emergencies and strengthened the health authority of the ministry to implement responses in case of risks or service discontinuity resulting from a lack of regional or local government compliance with public health functions.
\end{abstract}

Key words: Health care reform; Universal health coverage; Response to sanitary risks (source: MeSH NLM).

\section{INTRODUCCIÓN}

La Reforma del Sector Salud (1) se inició en el Perú en el año 2013 enfrentando un sistema que estaba fragmentado ${ }^{(2,3)}$, segmentado ${ }^{(4,5)}$, con insuficiente inversión (6-8), elevado gasto de bolsillo en salud ${ }^{(9,10)}$, bajo financiamiento ${ }^{(2)}$, deficiente acceso a servicios de salud, distribución inequitativa de recursos humanos, bajas remuneraciones ${ }^{(9)}$ y población vulnerable sin cobertura de algún seguro ${ }^{(3)}$. Además, la descentralización puesta

\footnotetext{
Universidad ESAN. Lima, Perú.

Ministerio de Salud. Lima, Perú.

Escuela de Medicina, Universidad Peruana de Ciencias Aplicadas. Lima, Perú.

Consejo Nacional de Salud. Lima, Perú

a Ministro de Salud, médico cirujano, magíster en Malariología y Saneamiento Ambiental; ${ }^{\text {a }}$ abogada, especialista en Derechos Humanos; ${ }^{\mathrm{c}}$ médico cirujano, especialista en Epidemiología de Campo.

Recibido: 18/05/2016 Aprobado: 20/07/2016
}

Citar como: Velásquez A, Suarez D, Nepo-Linares E. Reforma del sector salud en el Perú: derecho, gobernanza, cobertura universal y respuesta contra riesgos sanitarios. Rev Peru Med Exp Salud Publica. 2016;33(3)546-55. doi:10.17843/rpmesp.2016.333.2338 
en marcha desde el 2002 limitaba a la autoridad sanitaria nacional a funciones normativas y de supervisión no vinculante ${ }^{(3,12)}$, afectando la salud colectiva ${ }^{(13,14)}$ tal como se ha reportado en otros países ${ }^{(15,16)}$.

La Reforma se realizó siguiendo los principios de la Constitución Política del Perú que considera a la salud como un derecho e impone al Estado realizar acciones necesarias para lograr que todas las personas tengan acceso a los servicios de salud en condiciones de equidad y calidad. Es decir, que los ciudadanos puedan acceder a la protección social en salud, y a un nivel de vida que asegure su bienestar y brinde iguales oportunidades para el despliegue de sus capacidades ${ }^{(17)}$.

En el diseño de la Reforma participó el Consejo Nacional de Salud (1), así como funcionarios y expertos nacionales y extranjeros, en dos conferencias internacionales en los años 2013 y $2014{ }^{(18)}$. El Acuerdo Nacional, en el cual se proponen políticas de Estado, debatió la Reforma en el 2015, luego del cual se elaboró un consenso denominado "Objetivos de la Reforma de Salud" (19) (Figura 1).

Los 23 decretos legislativos (D.L.) que son base de la Reforma fueron autorizados mediante la Ley 30073, que otorgó facultades legislativas al poder ejecutivo.
Los objetivos principales fueron el fortalecimiento de la rectoría y gobernanza del sistema de salud, la protección de la salud individual, la protección de la salud colectiva, y la protección de los derechos en salud.

\section{MEDIDAS PARA FORTALECER LA GOBERNANZA Y RECTORÍA DEL SISTEMA DE SALUD}

En el 2002 se inició la descentralización del Estado con la transferencia de facultades a los gobiernos regionales; hasta el 2006 la estrategia fue de transición progresiva de funciones y facultades. En ese año se aceleró el proceso eliminando el requisito de acreditación (20). La descentralización del sector salud se realizó entre los años 2005 y 2009, quedando pendiente la transferencia al gobierno de Lima Metropolitana ${ }^{(21)}$. Con la descentralización de la función salud a los gobiernos locales, los gobiernos regionales se convierten tanto en la autoridad sanitaria como en los prestadores de servicios de salud en su ámbito geográfico, proceso que ha cambiado la organización administrativa regional, más no a nivel nacional, hasta la Reforma en Salud. Mediante el D.L. N 1161 se aprobó la Ley de Organización y Funciones (LOF) del Ministerio de Salud (MINSA) que detalla las competencias sectoriales de salud a nivel nacional

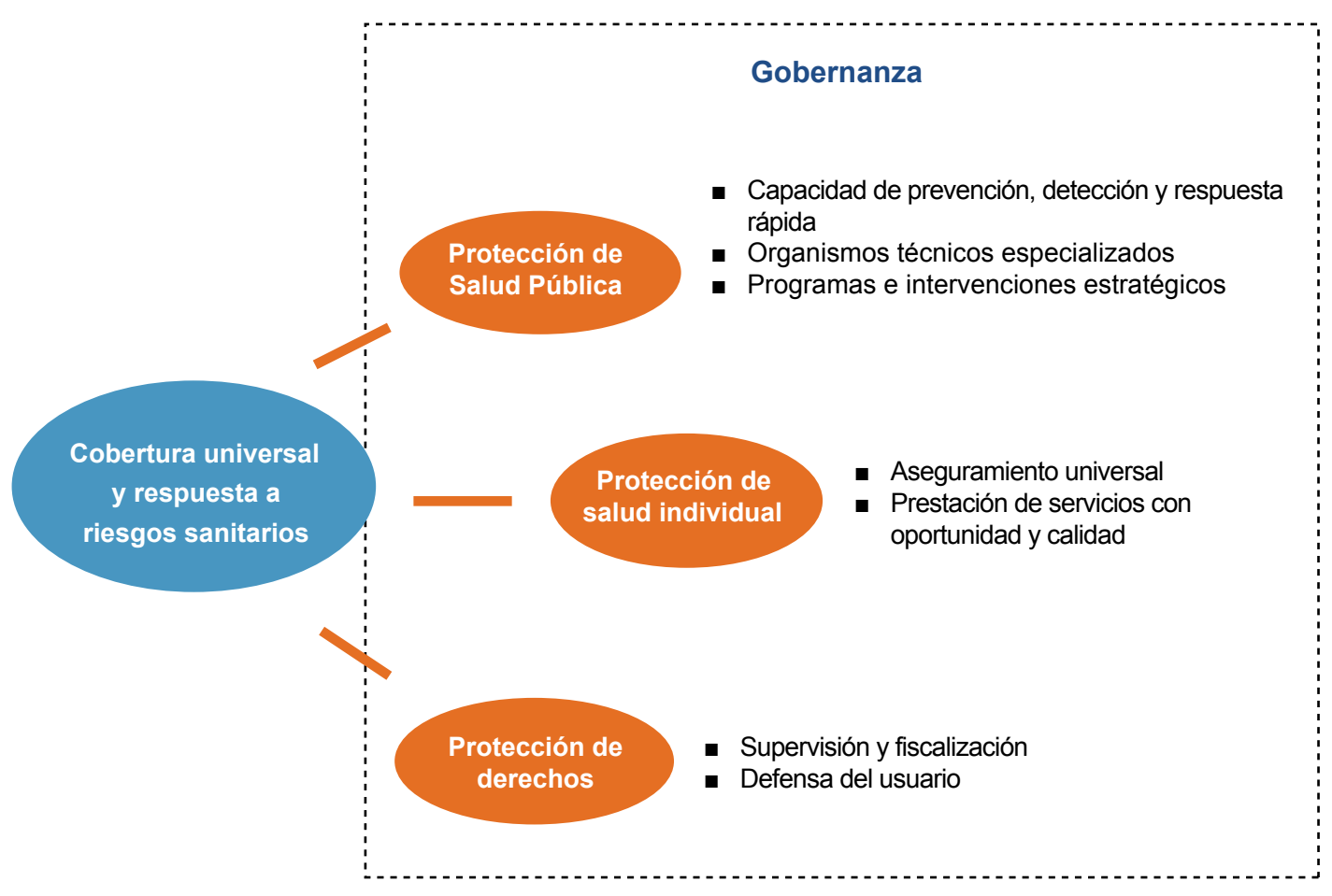

Fuente: Adaptado de Lineamientos y medidas de Reforma del Sector Salud. Lima: Consejo Nacional de Salud; 2013

Figura 1. Objetivos de la Reforma del sector Salud en el Perú 
acorde con el sistema descentralizado de salud. Posteriormente, el reglamento de la Ley se publicó en el año 2016 mediante Decreto Supremo 007-2016-SA. Se delimitan así las funciones exclusivas, las compartidas y otras específicas, lo cual fortaleció su rol rector.

El conjunto de normas de la Reforma busca fortalecer la capacidad regulatoria del MINSA, con el fin de mejorar el funcionamiento del sistema y el acceso a los servicios de salud; mejorar la capacidad de conducción del MINSA, al concertar con las autoridades sanitarias regionales alcances específicos de las normas; mejorar la modulación de la prestación para incrementar el uso de los recursos disponibles, la expansión planificada, y la organización de las redes de servicios; finalmente, la modulación del financiamiento, fortaleciendo el Seguro Integral de Salud (SIS) que puede comprar servicios al sector público y privado, y así financiar prestaciones complementarias y el intercambio de servicios entre prestadores; por otra parte, a las funciones rectoras del MINSA, según la Organización Panamericana de la Salud $^{(3)}$, se le ha añadido la respuesta nacional frente a riesgos y emergencias sanitarias.

Una característica particular del proceso de descentralización del sector Salud es el funcionamiento de la Comisión Intergubernamental de Salud (CIGS) que está encargada de desarrollar los componentes de la gestión, necesarios para que se ejerzan plenamente las funciones transferidas a los gobiernos regionales.

La CIGS, reunida trimestralmente desde el 2010, está conformada por el ministro de Salud y los principales representantes de las direcciones o gerencias regionales de salud (DIRESA o GERESA), y es un espacio de concertación sobre temas prioritarios del sector.

La CIGS ha permitido la implementación de políticas específicas, como la planificación de inversiones, por medio de los Comités Regionales Intergubernamentales de Inversión en Salud (CRIIS), cuya labor se ha materializado en la aprobación y priorización de los proyectos de construcción de 748 establecimientos estratégicos ${ }^{(20)}$. Las CRIIS elaboran el planeamiento multianual de inversiones regionales y las carteras anuales de inversión, y están formadas por el gobernador regional, los alcaldes provinciales y la autoridad sanitaria regional como secretaría técnica.Sesionan dos veces al año como mínimo.

Asimismo, con el D.L. 1167 se crea el Instituto de Gestión de Servicios de Salud (IGSS) como un organismo público adscrito al MINSA, especializado en la gestión prehospitalaria y hospitalaria de los servicios públicos de salud de Lima Metropolitana, separando la función rectora de la prestadora de servicios de salud del MINSA. Durante la implementación de la Reforma, el Perú estuvo expuesto a amenazas sanitarias y climáticas, como el fenómeno EI Niño, en medio de un sistema descentralizado de salud que requería ajustes para tener una acción eficaz del Estado y poder asegurar recursos para atender las situaciones de riesgos, epidemias y desastres; prevenir, mitigar sus potenciales daños y controlar sus efectos. Por eso, para fortalecer la autoridad de salud a nivel nacional y garantizar la prevención, control de riesgos y enfermedades de la población ${ }^{(22)}$ se promulgó la Ley 30423 que faculta al MINSA a "intervenir, mediante asistencia técnica, acompañamiento y movilización de recursos, en todo o parte del territorio nacional, por razones de necesidad de salud pública, emergencias sanitarias o desastres" (23). La norma se complementa con el D.L. 1156, que regula el procedimiento para la declaración de emergencias sanitarias. Su aplicación ha permitido realizar transferencias a los gobiernos regionales para enfrentar emergencias sanitarias.

Por otro lado, se ha creado recientemente la Comisión Sectorial de Presupuesto Multianual que reúne a los titulares de los pliegos presupuestales nacionales de salud adscritos al MINSA y la Junta Directiva de la Asamblea Nacional de Gobiernos Regionales (ANGR), cuyo propósito es establecer una mirada estratégica de las prioridades y el presupuesto del sector, correspondiendo que los gobernadores regionales convoquen al próximo titular del sector para presentarle las prioridades del presupuesto 2017-2019.

\section{MEDIDAS PARA LA PROTECCIÓN INDIVIDUAL: ASEGURAMIENTO EN SALUD Y FORTALECIMIENTO DE LA OFERTA}

\section{MEJORAR LA COBERTURA Y EL PAQUETE DE BENEFICIOS}

La cobertura de aseguramiento se ha incrementado a través del D.L: 1164, incorporando diversos grupos que habían quedado fuera del sistema de protección social en salud. Se complementa con la Ley 29951, que autoriza afiliar a independientes pequeños contribuyentes del Nuevo Régimen Único Simplificado (RUS), y con la Ley 30061, que declara de prioritario interés nacional la atención integral de la salud de los estudiantes adscritos al Programa Nacional de Alimentación Escolar Qali Warma. Adicionalmente, mediante el D.S. 010-2013-SA, se aprobó un Plan de Salud Escolar (Tabla 1). 
Tabla 1. Resultados en aseguramiento en salud

Resultados del aseguramiento en salud

Cobertura a 17,1 millones de peruanos para junio de 2016, lo cual representa un incremento de 32\% de población asegurada al SIS respecto al $2011^{*}$

65,9 millones de atenciones realizadas en el año $2015 ; 63,3 \%$ más que las realizadas en $2013^{\dagger}$

38,4 millones de atenciones de carácter preventivo realizadas a asegurados del SIS en $2015 ; 56,5 \%$ más que las realizadas en $2013^{\ddagger}$

436 mil afiliados al SIS, a setiembre 2015, mediante el Plan SIS Emprendedor, 61 mil más que en $2014^{\S}$

2,8 millones estudiantes de educación básica de Qali warma afiliados al SIS en 2015; 1,9 millones más que en 2014§

SIS: Seguro Integral de Salud

* Población asegurada 2016. † Población atendida 2013-2015. ^ Atenciones realizadas 2013-2015 (disponibles en: http://salasituacional. sis.gob.pe/Dashboard.aspx). § Sustentación del proyecto de presupuesto 2016 del sector salud (disponible en: http://goo.gl/6YVZOF).

De acuerdo a los datos de la Encuesta Nacional de Hogares, el aseguramiento en la población peruana se incrementó de 61,8 a $73,3 \%$ durante el periodo 2012 2015 (Figura 2). En esta misma encuesta, para elperiodo 2011-2015,el aseguramiento en población pobre subió de 62 a $74 \%$ y en población extremadamente pobre de 75 a $81 \%$. Según datos de la Superintendencia Nacional de Salud (SUSALUD), a la fecha, son 25 millones 961 mil 557 personas las que tienen algún seguro de salud $\left(82,45 \%\right.$ de la población) ${ }^{(24)}$.

Desde el año 2015, tanto recién nacidos como gestantes son afiliados automáticamente al Seguro Integral de Salud
(SIS) en caso no cuenten con otro servicio similar ${ }^{(25)}$. Dicha protección brinda servicios preventivos y curativos al recién nacido hasta los 5 años, incluyendo atenciones altamente especializadas y costosas. En el año 2015 se afiliaron al SIS más de 520 mil recién nacidos.

Al eliminar la barrera económica se incrementa el acceso y uso de servicios de salud, en particular en los más pobres ${ }^{(26)}$. En tal sentido, dicha barrera ha disminuido de 25 a $10 \%$ a nivel nacional, y si comparamos el periodo 2004 - 2014 en zonas pobres como en Apurímac, ha pasado de 39 a $3 \%$ (Figura 3 ).

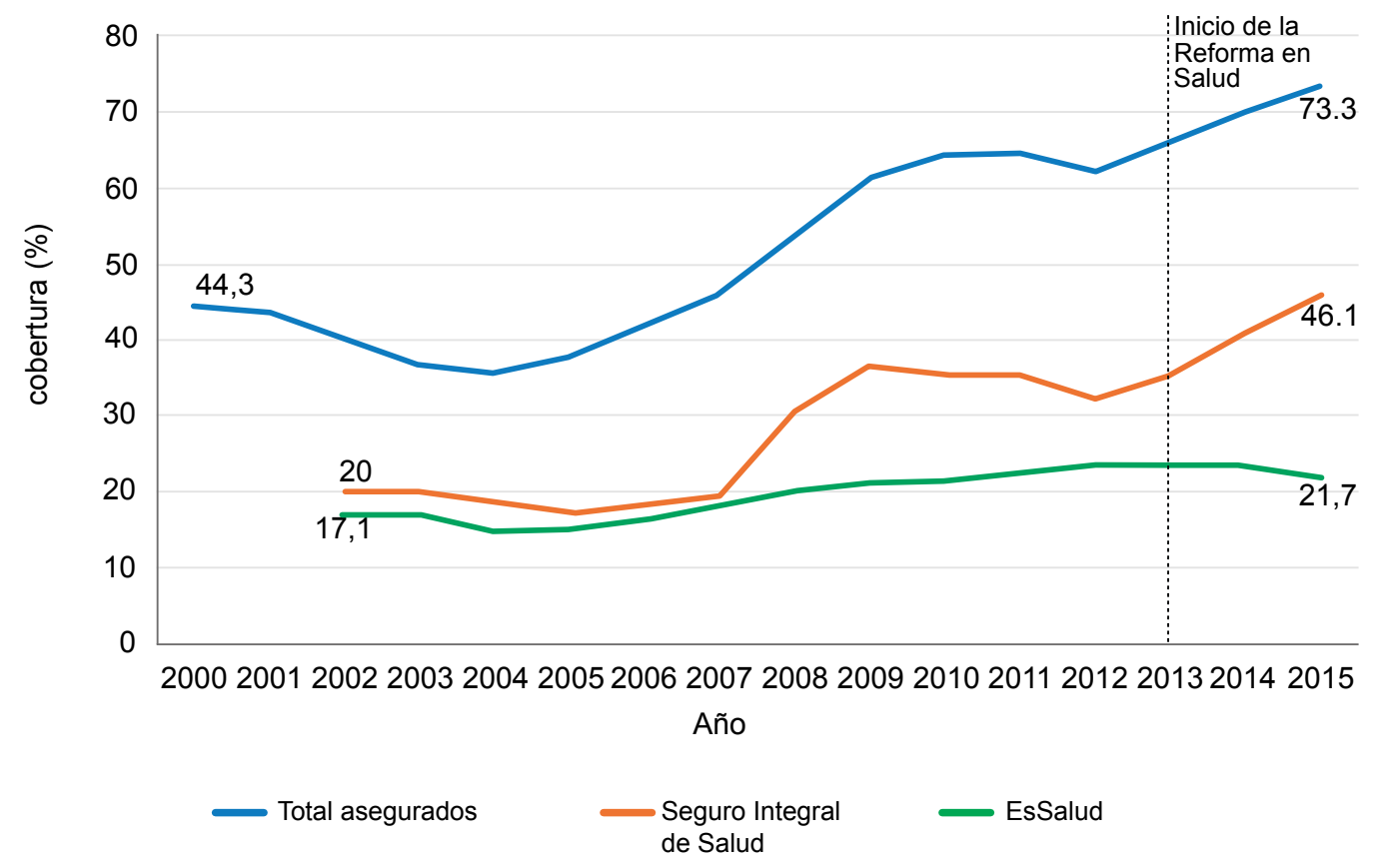

Fuente: Encuesta Nacional de Hogares 2000-2015

Figura 2. Evolución de la cobertura de aseguramiento en Salud en el Perú, 2000-2015. 


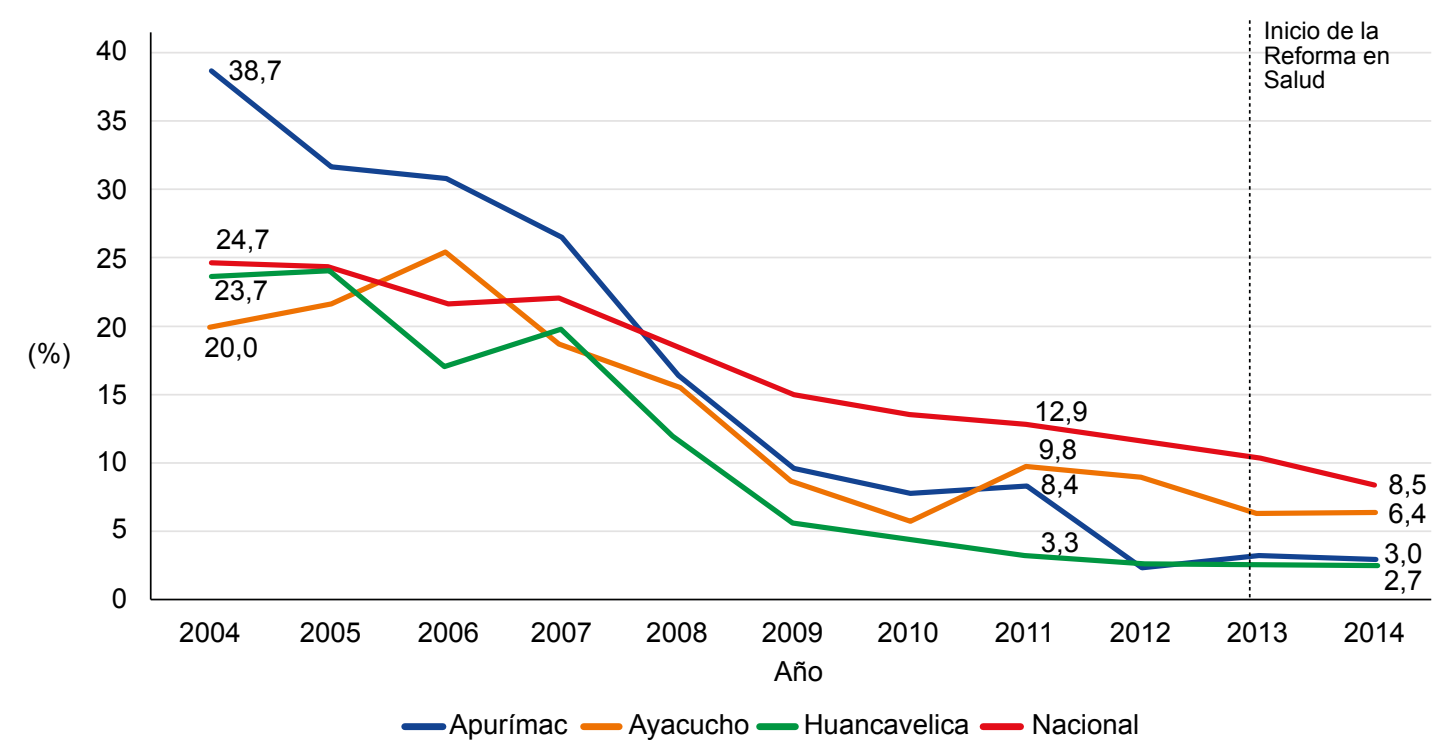

Fuente: Encuesta Nacional de Hogares 2000-2015

Figura 3. Falta de dinero como causa para no acudir a servicios de salud en tres regiones pobres del Perú

Además de incrementar su cobertura, el SIS amplió sus beneficios. Ambas mejoras se correspondieron con un incremento en su presupuesto, que se triplicó de 570 millones el año 2011 a cerca de 1700 millones de soles el año 2015.

La Reforma permitió mejorar el paquete de beneficios para los afiliados al SIS. Previamente, mediante la Ley de Aseguramiento Universal del año 2009 se creó el Paquete Esencial de Aseguramiento en Salud (PEAS) que incluía una lista priorizada de condiciones asegurables, intervenciones en salud, prestaciones o procedimientos de salud y garantías explicitas de oportunidad y calidad, que fue complementado por el Fondo Intangible Solidario de Salud (FISSAL) para cubrir las condiciones de alto costo (27). EI PEAS priorizaba implícitamente a mujeres embarazadas y niños -56 de 140 condiciones cubiertas eran obstétricas, ginecológicas y pediátricas- e incluía prestaciones en los tres niveles de atención, además que cubría los gastos de medicamentos que estuvieran incluidos en el Petitorio Nacional Único de Medicamentos Esenciales. La población afiliada al SIS sin capacidad de pago, era cubierta por el PEAS y el LPIS (Listado Priorizados de Atenciones en Salud), y pagaba a los establecimientos del MINSA por las exclusiones y límites en el uso.

Mediante el D.L. 1163, y una resolución jefatural del año 2012, se dispuso la ampliación del paquete de beneficios -que ahora incluye al PEAS y el plan complementario- y cobertura de emergencias. El plan complementario financia prestaciones no consideradas en el PEAS hasta un tope de dos unidades impositivas tributarias (UIT, USD 2293, aproximadamente) para prestaciones administrativas de traslado de emergencia, asignación por alimentación y gastos de sepelio. Además, se ha incluido un conjunto de prestaciones sujetas a autorización previa, denominado cobertura extraordinaría, que sirve para aquellos casos en el que la prestación de salud sobrepase las dos UIT, siempre que estas no representen exclusiones específicas como aquellas que no tengan como fin mejorar la salud $y / o$ tengan otra fuente de financiamiento (28).

EI SIS ahora transfiere recursos a los gobiernos regionales a través del pago capitado para la atención en el primer nivel mediante compromisos de gestión y pago por servicios prestados en los hospitales; si el paciente requiere ser atendido en servicios de mayor complejidad, en especial en áreas rurales de selva y sierra, el SIS financia el traslado con un acompañante a hospitales regionales y de Lima.

Con la Reforma se otorgó al SIS la capacidad para comprar servicios al sector privado, si es que no hay un servicio público que resuelva su demanda de salud. Con ello se supera la segmentación del sistema y se consolida un administrador de fondo que está protegiendo el derecho a la salud, sin importar quién brinde el servicio. Entre el 2012 y el 2016 se han realizado 95 mil prestaciones en servicios privados financiados por el SIS (Reporte de liquidación a terceros de la Gerencia de Negocios y Financiamiento 2012-2016).

En noviembre de 2012 se aprobó el Plan Esperanza, que otorga cobertura a enfermos de cáncer, con financiamiento del FISSAL ${ }^{(29)}$, en quimioterapia, 
radioterapia, procedimientos quirúrgicos y hospitalización. Este programa fue creado para prevenir y tratar el cáncer, y ha realizado desde el 2012 más de 7,8 millones de consejerias, más de 4,7 millones de personas han sido tamizadas y más de 122 mil personas han sido diagnosticadas y tratadas gratuitamente. Esto se amplió con el servicio de Esperanza Móvil que actúa de manera itinerante para detectar precozmente casos de cáncer.

Para los casos de leucemia y linfomas en menores de edad, siempre que el médico lo indique, SIS-FISSAL financia el trasplante de médula ósea en Lima y en el extranjero. Se han realizado más de 60 trasplantes de médula ósea, de los cuales ocho se hicieron fuera del país.

EI SIS, con la Reforma, financia la atención de escolares de educación inicial y primaria de escuelas públicas en áreas pobres junto al programa de alimentación escolar Qali Warma para mejorar el estado nutricional, prevenir y tratar la anemia, evaluar agudeza visual y entregar lentes correctores, vacunar niñas para prevenir cáncer de cervix, brindar atención de salud mental y realizar prevención y tratamiento de caries.

\section{MEJORAR LA GESTIÓN DE LAS INVERSIONES Y GENERAR MAYOR FINANCIAMIENTO}

La existencia de brechas de acceso a los servicios de salud se debe a diversos factores: déficit de recursos humanos ${ }^{(1)}$, limitaciones de infraestructura y equipamiento ${ }^{\left({ }^{30}\right)}$, debilidades en la gestión de la inversión en salud ${ }^{(31)}$, entre otros. El Estado durante muchos años dejó de invertir en establecimientos de salud, aun cuando la mayoría de hospitales tienen una antigüedad mayor de 50 años. Adicionalmente, existe una insuficiente cantidad de establecimientos con capacidad resolutiva básica y de hospitales de nivel intermedio.

Por otra parte, la modernización de la infraestructura y equipamiento no son sencillos, pues requieren de un sistema complejo de gestión de la inversión que considere a la numerosa cantidad de instituciones involucradas (gobierno nacional y locales), así como las fases del proceso (planeamiento, diseño, ejecución y evaluación de la inversión), en el que el Ministerio de Economía actúa como el rector del sistema de inversiones.

Las medidas de reforma estuvieron dirigidas a reducir las brechas entre la oferta y la demanda efectiva de los servicios de salud a nivel nacional, brindando servicios accesibles geográficamente y con capacidad resolutiva para una atención oportuna y de calidad.

Estas medidas regulan: el planeamiento multianual e intergubernamental de las inversiones para expandir la oferta, reestructurándola hacia la atención primaria de salud y construyendo el segundo nivel de atención, prácticamente inexistente en sector público; la reducción de la fragmentación de los prestadores de servicios de salud, facilitando el intercambio prestacional; el uso de la capacidad instalada ociosa de la oferta del sistema, mediante la extensión de horarios y estrategias de atención móvil, particularmente de atención especializada para hacer frente al embalse de cirugías programadas; la implementación de políticas de remuneraciones que fomenten una distribución más equitativa del personal de salud en el país y premie el esfuerzo de los trabajadores por brindar una atención de mayor calidad y oportunidad.

EI D.L. 1157 del año 2013, marco de la política sectorial de inversiones, establece condiciones para la modernización de la gestión de la inversión en salud, y determina los espacios de concertación intergubernamental como el CRIIS. Bajo el liderazgo del MINSA se ha desarrollado el plan multianual e intergubernamental 2016-2026 que contempla la construcción y puesta en operación de 468 establecimientos en el período 2016-2026, con una ampliación de la oferta que estará centrada en el primer y segundo nivel de atención (Tabla 2).

Esta planificación multianual permitió comprometer 8 mil 400 millones de soles para financiar la inversión de los establecimientos priorizados durante 2013 a 2016. Este fondo es administrado por el MINSA y puesto a disposición de los gobiernos regionales para financiar la construcción de establecimientos priorizados en los CRIIS.

Tabla 2. Proyectos de inversión en salud 2011 - 2016

\begin{tabular}{lccccc}
\hline & $\begin{array}{c}\text { Hospitales } \\
\text { nacionales }\end{array}$ & $\begin{array}{c}\text { Hospitales } \\
\text { regionales }\end{array}$ & $\begin{array}{c}\text { Hospitales provinciales } \\
\text { (segundo nivel de atención) }\end{array}$ & $\begin{array}{c}\text { Centros y puestos de salud } \\
\text { (primer nivel de atención) }\end{array}$ & $\begin{array}{c}\text { Total } \\
\text { (segulm }\end{array}$ \\
\hline Culminados & 2 & 6 & 21 & 139 & 168 \\
Por culminar & 0 & 4 & 7 & 24 & 51 \\
En proceso & 9 & 24 & 148 & 112 & 265 \\
\hline Total & 11 & 34 & & 275 & 468 \\
\hline
\end{tabular}


Los gobiernos regionales deben elaborar los perfiles de inversión para la creación de establecimientos priorizados y que, de ser viables, podrán acceder a recursos para las siguientes fases (expediente técnico, ejecución de la obra, equipamiento y operación). En la Ley de Presupuesto Anual se incluyen los montos que el MINSA transferirá a las regiones según nivel de avance de cada proyecto. Esta práctica permite un crecimiento más ordenado de la oferta y mejora la ejecución ya que la transferencia de recursos ocurre cuando se cumplen los estándares técnicos que el MINSA establece.

EI MINSA, a través del Programa Nacional de Inversiones en Salud (PRONIS, por Decreto Supremo 035-2014$\mathrm{SA})$, puede hacerse cargo del proyecto, construcción, equipamiento y supervisión de la obra, mediante convenio con el gobierno regional que no pueda realizar dicha función. Asimismo, el PRONIS tiene la función de supervisar la ejecución de los proyectos de inversión, participando activamente en la Comisión Multisectorial de Inversión en Salud (CMIS) y en los CRISS.

Durante periodo 2011-2016 se han puesto en operación 168 establecimientos de salud, 51 están por culminar y existen 265 proyectos que están en fase de expediente técnico o continuidad de obra (Tabla 2); en total significan una inversión ejecutada de más de 7 mil millones de soles. Además, se deja comprometida una cartera de proyectos de inversión de más de 13 mil millones de soles para los siguientes 3 años.

La política de inversiones considera, además, la puesta en operación del establecimiento (contratación de personal, mantenimiento, reposición de equipos, insumos, y medicamentos). Durante el periodo 2013 a 2016 se han transferido más de 188 millones de soles para equipar y mantener los servicios y más de 100 millones para contratación de personal de establecimientos recién inaugurados.

Para acelerar la inversión, la Ley 30167 permite la participación del sector privado mediante Asociaciones Público Privadas (APP) y obras por impuestos para el diseño, construcción, equipamiento, mantenimiento y operación de servicios generales. Para el año 2016 se iniciará la construcción de tres hospitales mediante esta modalidad, por un monto de 237 millones de soles. Además, ya están en formulación proyectos que beneficiarán a varios hospitales de Lima, Sullana y Piura, con un monto de inversión de 4150 millones de soles. A su vez, con esta modalidad se ha financiado la construcción una planta de tratamiento de residuos hospitalarios por un monto de 117 millones de soles.

\section{AUMENTAR LA DOTACIÓN DE RECURSOS HUMANOS Y MEJORAR SU REMUNERACIÓN}

La Reforma puso énfasis en mejorar las remuneraciones del personal asistencial. Los D.L. 1153 y 1162 permitieron nivelar las remuneraciones de los trabajadores del interior del país con los de la capital. También se creó un sistema de compensaciones destinadas a dar incentivos para el trabajo especializado, en áreas críticas, en atención primaria de salud, en zonas alejadas y de frontera, zonas de emergencia, por responsabilidad de jefatura y al buen desempeño. A partir de la vigencia del D.L. 1153, se incrementó el presupuesto para remuneraciones de 1500 millones de soles en 2013 a 2 350 millones en $2016^{(32)}$.

Así, el número de médicos se incrementó de 26762 en el año 2010 a 38065 en el 2015; enfermeros de 29625 a 39 979, y obstetras de 9882 a 14 445. De acuerdo al Registro Nacional del Personal de la Salud en el Perú $2015^{(33)}$, en nuestro país existe una densidad de 12,2 médicos, 12,8 enfermeros, y 4,6 obstetras por cada 10 mil habitantes, cifras que nos ubican por encima de los estándares de la OMS para países de ingresos medianos bajos ${ }^{(34)}$.

Además, las plazas para formación de especialistas aumentaron de 1495 a 3212 desde el año 2011. Se cuenta hoy con una Ley 30453 de Residentado Médico que, junto con la política de desarrollo de recursos humanos, permitirá incrementar la formación de residentes y generar incentivos para que luego de concluir sus estudios vayan a trabajar en las regiones deficitarias.

\section{MAYOR ARTICULACIÓN Y EFICIENCIA DEL SISTEMA DE SALUD}

La Reforma también permite el intercambio prestacional -especialmente entre el MINSA y el Seguro Social de Salud (EsSalud)-, para que las administradoras de fondos puedan pagar por servicios prestados a sus afiliados. Entre el 2012 y el 2015 se han realizado más de 52 mil atenciones de afiliados de ESSALUD en establecimientos públicos, fundamentalmente de baja complejidad, y más de 2 mil atenciones de afiliados del SIS en EsSalud, básicamente de mediana y alta complejidad.

Se empezó a aplicar un nuevo enfoque de entrega de servicios especializados a través del Plan Más Salud, que se caracteriza por enviar entre 20 a 30 especialistas a las regiones con más déficit de atención médica para realizar campañas médico-quirúrgicas sobre demanda programada. Más Salud ha llegado a todas las regiones 
y ha realizado más de un millón de atenciones, más de 1432 cirugías, 21534 procedimientos de diagnóstico y tratamiento especializado, incluyendo cirugías al corazón, intervenciones endoscópicas y el uso de equipamiento de última generación.

La Reforma incluyó la aprobación de la Ley de Telemedicina y Telesalud (Ley 30421). Actualmente, el MINSA está implementando un servicio de telemedicina desde hospitales de Lima, cuyos especilistas serán remunerados por el SIS, para dar asistencia en tiempo real a pacientes que se encuentran en hospitales de La Libertad, Arequipa, Junín, Loreto, Cajamarca, Ucayali, Huánuco y Amazonas. Este servicio mejorará notablemente con la culminación de la red dorsal de fibra óptica que llegará a todas las provincias del país.

La Reforma busca un mayor acceso a los medicamentos. El Centro Nacional de Abastecimiento de Recursos Estratégicos en Salud actúa como una central de compras corporativas y desarrolla un sistema logístico de distribución efectiva. Otra iniciativas incluyen la entrega a usuarios SIS, de medicamentos en farmacias privadas, la creación de farmacias institucionales para la venta de genéricos, farmacias gratuitas para afiliados al SIS, y el desarrollo estudios de intercambiabilidad para otorgar garantía de seguridad y eficacia de medicamentos.

Por otro lado, en el mismo contexto, se dictaron normas para adecuar la organización de los Fondos de Salud de las Fuerzas Armadas y Policía Nacional, a las disposiciones normativas vigentes que rigen el Sistema Nacional de Salud (D.L. 1173, 1174 y 1175). Adicionalmente, se dictaron medidas para mejorar la relación con la Superintendencia Nacional de Aduanas y de Administración Tributaria (SUNAT) y EsSalud con los D. L. 1160, 1168, 1170, 1171 y 1172.

\section{MEDIDAS PARA PROTEGER LA SALUD COLECTIVA}

La modificación de los mecanismos de pago del SIS, parte de la Reforma, permitió complementar la modulación de su financiamiento, y ha logrado que las atenciones preventivas superen a las atenciones recuperativas (Figura 4), en base al pago capitado que incentiva el cumplimiento de metas en el primer nivel de atención sobre prioridades de salud materna e infantil, prevención de enfermedades transmisibles y crónicas.

Estas metas y las reglas de financiamiento se establecen de mutuo acuerdo con los gobiernos regionales. Al

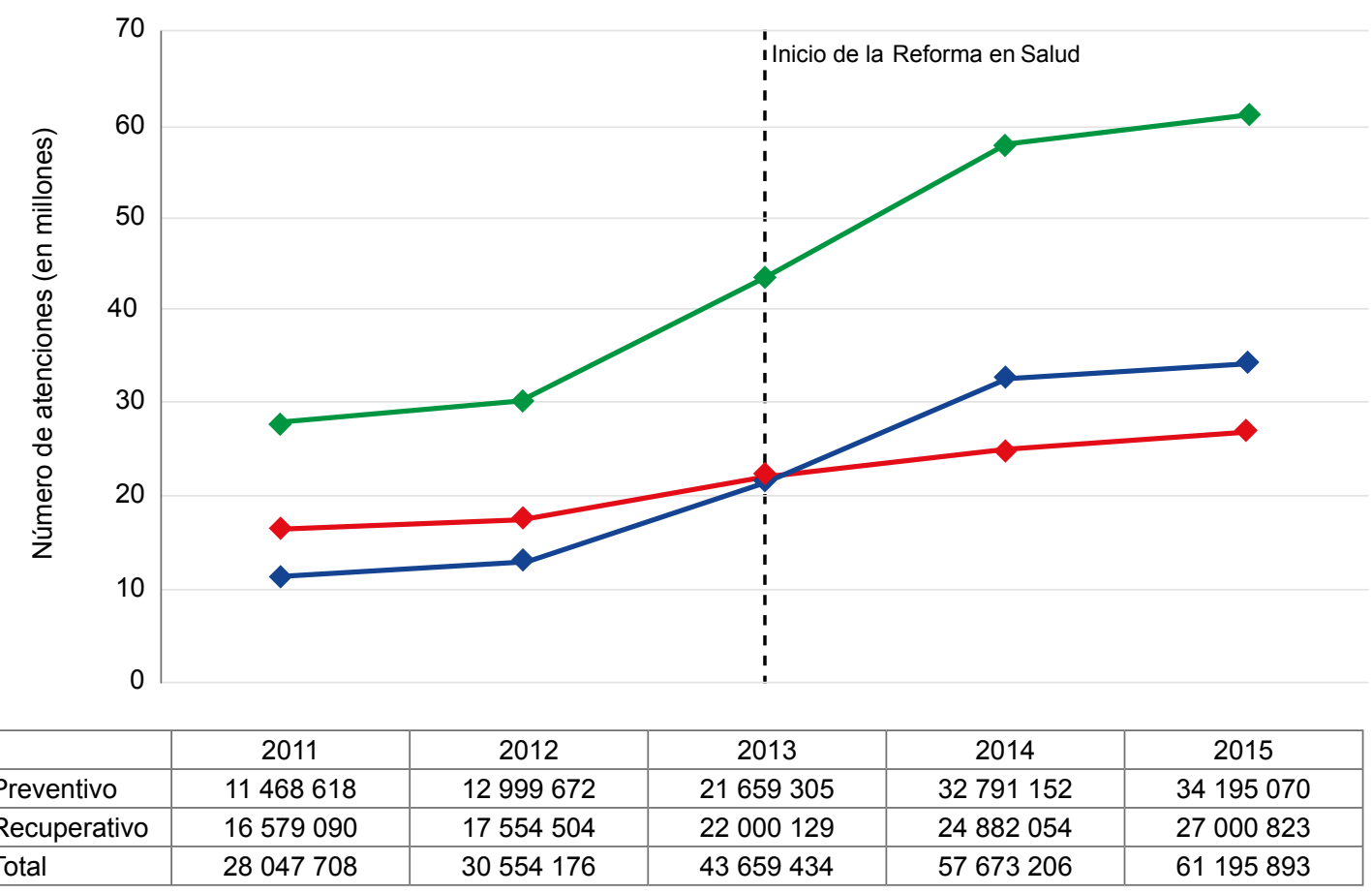

Fuente: Gerencia de Riesgos y Evaluación de Prestaciones. Seguro Integral de Salud.

Figura 4. Tipo de atención de salud brindada a asegurados del Seguro Integral de Salud 
inicio, el pago capitado se hace por adelantado, y los siguientes desembolsos se hacen siempre y cuando se cumpla con las metas.

Con la promulgación de la Ley 30423 y el actual Reglamento de Organización y Funciones del MINSA, se crea el Centro Nacional de Epidemiología, Prevención y Control de Enfermedades (CDC), considerado como el organismo especializado del MINSA con capacidad de intervenir en caso de riesgos o emergencias sanitarias. EI CDC agrupa unidades técnicas del MINSA que se encontraban repartidas en varias direcciones e institutos, como las denominadas estrategias sanitarias, los laboratorios de salud pública, vigilancia epidemiológica, vigilancia entomológica; y algunas nuevas como los equipos de respuesta rápida.

Durante el periodo de la Reforma, el Perú ha enfrentado la epidemia de dengue y enfermedades causadas por los virus de chikungunya y zika, para lo cual se declararon áreas en emergencia o en riesgo, se transfirieron recursos, se contrató personal, y se compró medicamentos, insumos y equipos. Asimismo, se nombraron altos comisionados del MINSA que, junto con autoridades regionales, formaron comisiones intergubernamentales y multisectoriales mientras duraba la emergencia. Los resultados de estas acciones, la vigilancia del vector y el manejo focalizado buscaron retrasar la transmisión autóctona de chikungunya. Para el año 2015 se registraron 186 casos en Tumbes y Piura; mientras que hasta julio de 2016, se tuvieron 152 casos. Esto contrasta con los miles de casos registrados en el continente.

Respecto al Zika, desde el 2014 en que se reportó transmisión autóctona en la región, Brasil identificó 72 596 casos y Colombia 10 016. En el Perú, el primer caso importado ocurrió en enero de 2016, y hasta julio del 2016 existen 86 casos autóctonos notificados.

Además, la Reforma permite que el MINSA maneje programas específicos para mejorar determinados indicadores prioritarios como son la mortalidad neonatal y la caries dental en escolares. Para el primer caso, con el Plan Bienvenidos a la Vida (se entrega una caja cuna con ropa y utensilios para el cuidado de más de 74 mil recién nacidos afiliados al SIS), o el Plan de Salud Dental Escolar que realiza actividades educativas, preventivas y curativas para proteger la salud bucal.

\section{MEDIDAS PARA LA DEFENSA DE LOS DERECHOS Y PARTICIPACIÓN DE LOS USUARIOS}

La Superintendencia Nacional de Salud (SUSALUD), se creó sobre la base de la Superintendencia Nacional de Aseguramiento en Salud (SUNASA), a través del D. L. 1158 para defender los derechos de las personas al acceso a los servicios de salud y supervisar la prestación de dichos servicios. En el 2015 ha atendido 19731 reclamos $^{(35)}$ y realizado 833 supervisiones a IAFAS e IPRESS públicas y privadas, y ha implementado un programa de delegados en 32 establecimientos de salud e instalado juntas de usuario en siete regiones del país ${ }^{(35)}$.

SUSALUD tiene funciones supervisoras y fiscalizadoras con capacidad sancionadora a las instituciones prestadoras de servicios de salud y a las instituciones administradoras de fondos de aseguramiento en salud.También tiene a su cargo informar y promover que los ciudadanos conozcan sus derechos y cómo hacerlos respetar, así como atender a los usuarios cuando se vulneren esos derechos.

A modo de conclusión podemos señalar que hemos avanzado en el proceso de implementación de la reforma, los logros están en su fase inicial. Este camino debe continuar para seguir dando sostenibilidad a políticas de salud que no son solo de un Gobierno sino de varios, lo que las hace convertirse en políticas de estado.

Contribuciones de autoría: AV, DS, ENL han participado en la concepción del artículo, la recolección de datos, su redacción y aprobación de la versión final.

Fuentes de financiamiento: autofinanciado.

Conflictos de interés: los autores declaran no tener conflicto de intereses.

\section{REFERENCIAS BIBLIOGRÁFICAS}

1. Consejo Nacional de Salud del Perú. Lineamientos y medidas de reforma del sector salud [Internet]. Lima: Consejo Nacional de Salud; 2013 [citado el 16 de mayo de 2016]. Disponible en: http://www.minsa.gob.pe/portada/ Especiales/2013/reforma/documentos/ documentoreforma11122013.pdf

2. Atun R, de Andrade LO, Almeida G, Cotlear D, Dmytraczenko T, Frenz P, et al. Health-system reform and universal health coverage in Latin America. Lancet. 2015;385(9974):1230-47. doi: 10.1016/S0140-6736(14)61646-9

3. Cetrángolo $\mathrm{O}$, Bertranou $\mathrm{F}$, Casanova L, Casalí P. El sistema de salud del Perú: Situación actual y estrategias para orientar la extensión de la cobertura contributiva. $1^{\text {a }}$ ed. Lima: OIT/ Oficina de la OIT para los Países Andinos; 2013
4. Alcalde J, Nigenda G, Lazo O. Financiamiento y gasto en salud del Sistema de Salud en Perú [Internet]. México: Fundación Mexicana para la Salud, A.C.; 2013 [citado el 16 de mayo de 2016]. Disponible en: http:// www.funsalud.org.mx/competitividad/ financiamiento/Documentos/ Working\%20paper\%202/Peru\%20 sistemico.pdf 
5. Alcalde-Rabanal JE, Lazo-González O, Nigenda G. Sistema de salud de Perú. Salud PUblica Mex. 2011;53(Supl 2):S243-S54.

6. Monje-Vargas JA. Equidad e inversión en salud pública en la República del Perú. Rev Cubana Salud Publica. 2011;37(4):452-61.

7. Huayanay-Espinoza CA, Huayanay A, Huicho L. Incremento en el gasto por inversión en el sector salud: jeficiencia y efectividad en el gasto? Rev Peru Med Exp Salud Publica. 2015;32(4):817-24.

8. Petrera M. Algunas reflexiones en torno a las cuentas nacionales de salud del Perú. Rev Peru Med Exp Salud Publica. 2009;26(2): 248-50.

9. Portocarrero-Grados A. Boletín $\mathrm{N}^{\circ}$ 2. El pago de bolsillo en el financiamiento de los servicios de salud. Lima: Consorcio de Investigación Económica y Social (CIES); 2005.

10. Lavilla-Ruiz H. Empobrecimiento por Gasto de Bolsillo en Salud. Incidencia del Gasto de Bolsillo en Salud en el Perú, 2006-2009. Lima: Consorcio de Investigación Económica y Social (CIES); 2012. p. 66

11. Perú, Ministerio de Salud. Recursos Humanos en Salud al 2011: Evidencias para la toma de decisiones. Dirección General de Gestión del Desarrollo de Recursos Humanos. Observatorio Nacional de Recursos Humanos en Salud. Lima: MINSA; 2011.

12. Mendoza-Arana P, Mostajo-Vallenas P, Velásquez-Valdivia A, Cotrina-Rabanal A, Jaramillo-Bahanante M. El Sistema Regional de Salud de San Martín y su política de reducción de la desnutrición infantil: aplicación en el Perú del Enfoque de Evaluación de Sistemas de Salud/Health Systems Assessment. An Fac med. 2015;76(3):269-76. doi: http://dx.doi.org/10.15381/anales. v76i3.11238.

13. La Contraloría General de la República de Perú. Estudio del proceso de descentralización en el Perú. Lima: La Contraloría General de la República de Perú; 2014. pp.11-19.

14. Cosavalente O. Sistematización del proceso deimplementación del monitoreo y evaluación de la descentralización en salud en el nivel regional orientado a mejorar el desempeño de la función Salud. Lima: MINSA/Programa de Apoyo a la Reforma del Sector Salud PARSALUD II; 2010.

15. Kalk A, Fleischer K. The decentralization of the health system in Colombia and Brazil and its impact on leprosy control. Lepr Rev. 2004;75(1):67-78.
16. Yadón ZE, Gürtler GE, Tobar F, Medicine AC. Descentralización y gestión del control de las enfermedades transmisibles en América Latina. Buenos Aires: OPS; 2006.

17. Naciones Unidas. Comité de Derechos Económicos, Sociales y Culturales. Observación General No 14 (2013) sobre el derecho del nińo a que su Interés superior sea una consideración primordial (artículo 3, párrafos 1). Ginebra: Naciones Unidades; 2013.

18. Perú, Ministerio de Salud. Hacia la Cobertura Universal en Salud. Lima: MINSA; 2015.

19. Acuerdo Nacional. 2016. Los objetivos de la reforma de la salud [Internet]. Lima: Acuerdo Nacional; 2015 [citado el 16 de mayo de 2016]. Disponible en: http://acuerdonacional.pe/2015/10/losobjetivos-de-la-reforma-de-salud/

20. De Habich M. Propuesta de medidas para la implementacion del consenso en acuerdo nacional: "Los objetivos de la reforma en salud". Lima: BID; 2016.

21. Távara G, Márquez J. Sistematización del proceso de descentralización del sector salud. Lima: USAID/PERÚ; 2009.

22. Perú, Congreso de la Republica. Ley que establece medidas para fortalecer la autoridad de salud de Nivel Nacional, con el fin de garantizar la prevención, control de riesgos y enfermedades de la población. Ley 30423. El Peruano. 19 de abril de 2016.

23. Perú, Ministerio de Salud. Decreto Legislativo que aprueba la Ley de Organización y Funciones del Ministerio de Salud. Decreto Legislativo 1161. El Peruano. 6 de diciembre de 2013.

24. Superintendencia Nacional de Salud. Sureporte. Boletín Informativo. Registro nominal de afiliados de SUSALUD al 26 de junio del 2016 [Internet]. Lima: SUSALUD; 2016 [citado el 16 de mayo de 2016]. Disponible en: http://portales.susalud. gob.pe/documents/11450/164131/1+Afiliados_270616.pdf/82b614d298df-4f4d-9426-0d5e1495bc2e

25. Perú, Ministerio de Salud. Plan Nacional "Bienvenidos a la Vida". En el Marco de las Acciones de Fortalecimiento para la Reducción de la Morbi-Mortalidad Neonatal en el Perú 2015-2016. Resolución Ministerial 997-2014/MINSA. El Peruano. 24 de diciembre de 2014.

26. Escobar ML, Griffin CC, Shaw RP. The Impact of Health Insurance in Low and Middle Income countries. Washington, D.C.: Brookings Institutions; 2010.
27. Prieto L, Cid C, Montañez V. Capítulo 5. Perú: el Plan Esencial de Aseguramiento en Salud. En: Giedion U, Bitrán R, Tristao I. Planes de beneficios en salud de América Latina. Una comparación regional. Washington, D.C.: Banco Interamericano de Desarrollo; 2014. pp. 170-98.

28. Seguro Integral de Salud, Perú, Ministerio de Salud. Directiva que regula la cobertura prestacional del regimen de financiamiento subsidiado del Seguro Integral de Salud. Directiva Administrativa 003-2012-SIS-GREP. (6 de diciembre de 2012).

29. Perú, Ministerio de Salud. Declaran de interés nacional la atención integral del cáncer y mejoramiento del acceso a los servicios oncológicos en el Perú y dictan otras medidas. Decreto supremo 0092012-SA. El Peruano. 3 de noviembre de 2012.

30. García-Cabrera HE, Diaz-Urteaga P, Ávila-Chávez D, Cuzco-Ruiz MZ. La Reforma del Sector Salud y los recursos humanos en salud. An Fac Med. 2015; 76:7-26.

31. Madueño M, Sanabria C. Estudio de oferta de los servicios de salud en el Perú y el análisis de brechas 2003-2020 (Documento 3) [Internet]. Meryland: PHRplus; 2003 [Citado el 01 de junio de 2016] Disponible en: http://www. phrplus.org/Pubs/Tech026s_fin.pdf

32. Ministerio de Economía y Finanzas del Perú. Consulta Amigable [Internet]. Lima: MEF; 2016 [citado el 12 de mayo de 2016]. Disponible en: http:// apps5.mineco.gob.pe/transparencia/ Navegador/

33. Dirección General de Gestión del Desarrollo de Recursos Humanos, Perú, Ministerio de Salud. Información de Recursos Humanos en el Sector Salud. Lima: Dirección General de Gestión del Desarrollo de Recursos Humanos/ MINSA; 2015.

34. Organización Mundial de la Salud. Estadísticas Sanitarias Mundiales 2014. Ginebra: OMS; 2014.

35. Velásquez A. Políticas y Acciones en Salud hacia el Bicentenario. Documento de Gestión 2015 [Internet]. Lima: MINSA; 2015 [citado en 01 de junio de 2016]. Disponible en: http://bvs.minsa.gob. pe/local/MINSA/3476.pdf

Correspondencia: Edgardo Nepo Linares

Dirección: Ismael Bielich 145, departamento 202. Santiago de Surco, Lima 33

Teléfono: (+511) 4480310

Correo electrónico: galonepo@gmail.com 